\title{
Brain lesion in a Wistar rat
}

\author{
Yogeshkumar V. Murkunde, MVSc ${ }^{1}$, Ponnusamy Kalaiselvan, MVSc ${ }^{1}$, Subramaniyan Vijayakumar, MVSc ${ }^{1}$, \\ Kuppusamy Hemalatha, MVSc ${ }^{1}$, Robert R. Maronpot, DVM, MS, MPH, DACVP, DABT ${ }^{2}$, Ronald A. Herbert, DVM, PhD \\ \& Monique Y. Wells, VMD, MS, DACVP, DECVP, DABT, ERT ${ }^{3}$
}

During a two-year carcinogenicity study on an agrochemical compound, histopathological evaluation showed necrosis and moderate inflammation in the brain of a female Wistar rat. The study was performed under Good Laboratory Practice (GLP) guidelines.

Four hundred and sixty Wistar/NIN rats (230 males and 230 females) were divided into four groups and housed five per cage in polypropylene cages with stainless steel grill lids under standard husbandry conditions (room temperature $18-21^{\circ} \mathrm{C}$, relative humidity $40-70 \%$, light:dark cycle of $12 \mathrm{~h}: 12 \mathrm{~h}$ ). They received small, round masses of feed (prepared by mixing the test substance with powdered diet from Amrut Laboratory Animal Feed, Pune, India) and filtered, ultraviolettreated water ad libitum. The test substance was administered at doses of 0, 10,000, 20,000 and 40,000 ppm over a 2 -year period. Rats were obtained from the International Institute of Biotechnology and Toxicology (Tamil Nadu, India) breeding colony.

Major clinical signs exhibited by the test animals included convulsions, general body weakness and piloerection. At the end of the treatment period, animals were euthanized by carbon dioxide asphyxiation. Each animal underwent a complete necropsy. Gross pathological findings were not remarkable; no notable macroscopic lesions were observed in the brain of any animal.

Protocol-required tissues and gross lesions were collected, fixed by immersion in $10 \%$ neutral buffered formalin and processed for histopathologic evaluation. Paraffinembedded tissues were sectioned at intervals of 3-4 $\mu \mathrm{m}$, mounted on glass slides and stained with hematoxylin and eosin.

Upon histopathologic evaluation of the brain, we observed perivascular cuffs or

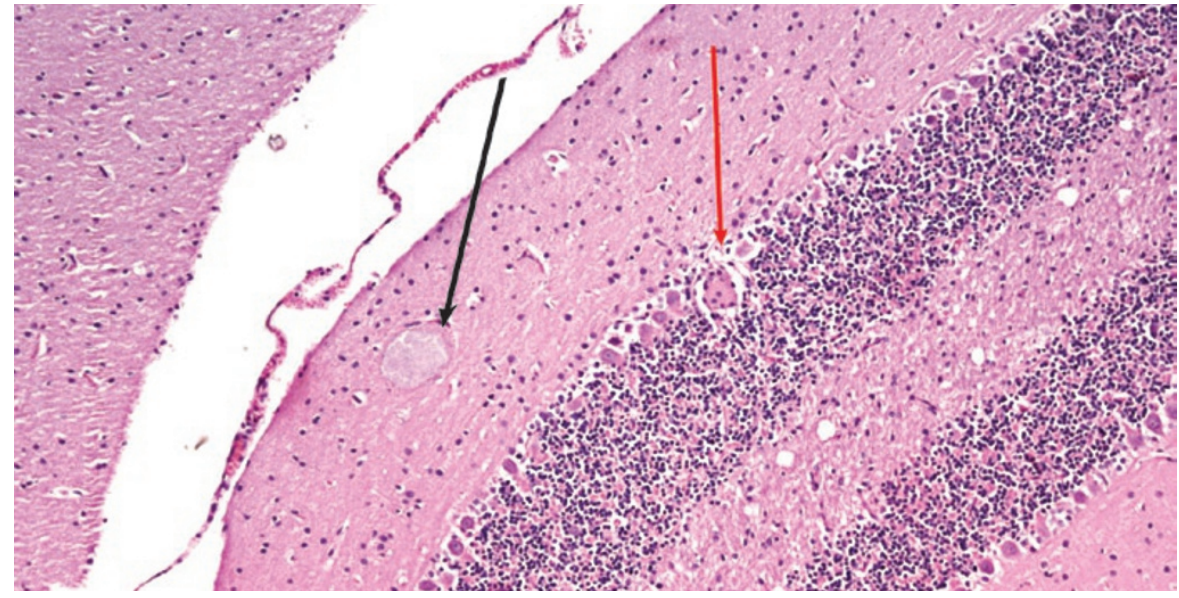

FIGURE 1 | Cerebellum of a high-dose female rat showing a pseudocyst in the molecular layer (black arrow) and a small granuloma (red arrow) at the junction of the Purkinje and granular layers. Hematoxylin and eosin staining; 10x magnification.

submeningeal mononuclear inflammatory cell infiltrate in the cerebrum, cerebellum or brainstem of 8 male and 10 female rats. We observed nonsuppurative (mononuclear and/or histiocytic) inflammation of brain parenchyma in 4 males and 8 females. All inflammatory lesions in these animals were minimal to mild in severity. Most lesions were observed in treated animals; we observed only one control animal with an inflammatory lesion in the brain (nonsuppurative inflammation). These lesions were not dose-related in incidence and were therefore considered to be spontaneous in nature.

One high-dose female rat had a large focus of necrosis, a submeningeal mononuclear cell infiltrate and moderate nonsuppurative inflammation in the cerebellum (Fig. 1). By careful evaluation of the parenchyma, we identified multiple pseudocysts in the molecular layer of the cerebellum, characterized by numerous small, rod-shaped organisms enclosed in a thin-walled membrane. Clinical signs and gross pathological findings were unremarkable for this animal. No other organ had such lesions.

Another high-dose female had minimal perivascular cuffing and mild nonsuppurative inflammation without associated pseudocysts in the spinal cord but not in the brain. Other histopathologic findings in the study were background changes that are commonly observed in rats of this strain and age.

What are the pseudocysts? Could the test substance have caused them? In what species might you expect to see the pseudocysts? Is the brain the organ where such pseudocysts are usually seen? Aside from histopathology, what techniques might you use to diagnose the pseudocysts?

\section{What's your diagnosis?}

${ }^{1}$ Department of Pathology, International Institute of Biotechnology and Toxicology, Padappai 601301, Kancheepuram District, Tamil Nadu, India. ${ }^{2}$ Cellular and Molecular Pathology Branch, National Institute of Environmental Health Sciences, Research Triangle Park, NC 27709 USA. ${ }^{3}$ Toxicology/Pathology Services Inc., 52 , rue Lhomond, 75005 Paris, France. Correspondence should be addressed to Y.V.M. (fippat@giasmd01.vsnl.net.in). 